\title{
Fase gametofítica de las tres variedades de Argyrochosma nivea (Pteridaceae)
}

\author{
Olga G. Martínez ${ }^{1}$ \& Marcela A. Hernández ${ }^{2}$ \\ ${ }^{1}$ Facultad de Ciencias Naturales-IBIGEO (CONICET), Universidad Nacional de Salta, Av. Bolivia 5150, \\ 4400 Salta, Argentina. \\ ${ }^{2}$ Fundación Miguel Lillo, M. Lillo 251, T4000JFE Tucumán, Argentina
}

\section{Correspondencia}

O.G. Martínez

e-mail: martinezog@gmail.com

Recibido: 1 julio 2017

Aceptado: 14 septiembre 2017

Publicado on-line: diciembre 2017

\begin{abstract}
Resumen
La finalidad de este trabajo es describir características de la fase gametofítica de Argyroschoma nivea con el objeto de caracterizar esta fase del ciclo de vida y aportar evidencias a las relaciones entre las tres variedades de la especie (var. flava, var. nivea, y var. tenera). Para ello se consideraron cantidad de esporas por esporangio, tamaño de las esporas y se obtuvieron los gametofitos mediante cultivos en medios estériles. Se realizaron observaciones mediante microscopía de luz y electrónica de barrido. Se encontró que las tres variedades presentan 32 esporas por esporangio, comparten idéntico patrón de germinación, desarrollan gametofitos cordiformes asimétricos, y su reproducción es apogámica, de tipo obligada en las var. nivea y tenera. Los esporofitos de la var. flava desarrollan tricomas secretores de farina de color blanco sobre toda la superficie.
\end{abstract}

Palabras clave: Ambientes xéricos, farina, gametofitos, cheilantoideos.

\begin{abstract}
Gametophytic phase of the three varieties of Argyrochosma nivea (Pteridaceae).
\end{abstract}

The purpose of this work is to describe the gametophytic phase of Argyroschoma nivea in order to characterize this phase of the life cycle and provide evidence for the relationships between the three varieties (var. flava, var. nivea and var. tenera). For this, spores were considered by sporangium, size of spores, and gametophytes were obtained by culture in sterile media. Observations were made using light microscopy and scanning electron microscopy. It was found that the three varieties present 32 sporangia spores, share the same germination pattern, develop asymmetric cordiform gametophytes, and their reproduction is apogamic, obligatory type in var. nivea and tenera. The sporophytes of var. flava develop glandular trichomes excretors white farina over of surface.

Key words. Farina; gametophyte; xeric environments, cheilanthoid.

\section{Introducción}

El género Argyrochosma (J. Sm.) Windham, de origen americano y según Sigel et al. (2011) monofiletico, reúne unas 20 sp., estos helechos constituyen parte de la diversidad de los helechos cheilantoides de la familia Pteridaceae, son plantas de ambientes xéricos a estacionalmente xéricos (Tryon et al., 1990; Windham, 1987). La especie Argyrochosma nivea con sus tres variedades (var. flava, var. nivea y var. tenera) crece en Sudamérica subtropical y las variedades se identifican por la ausencia de ceras o "farinas" en la var. tenera, y presencia de farina blanca en la var. nivea o 
amarilla en la var. flava (Ponce, 2016). Estas plantas saxícolas crecen entre las rocas, en sus grietas o sobre rocas cubiertas de líquenes.

Una de las adaptaciones de estas plantas es el exudado de farinas presentes en la superficie abaxial de la lámina donde son producidas por tricomas glandulares, proporcionando a las plantas protección contra la luz solar intensa y baja humedad, además reducen el movimiento del aire sobre la epidermis. Cuando la desecación del ambiente es prolongada, las láminas se envuelven sobre la superficie adaxial dejando expuesta la epidermis con farina (Hevly, 1963; Wollenweber, 1984).

En la taxonomía de los helechos, la información palinológica contribuye a la delimitación de los diferentes grupos, no solo la estructura y ornamentación de las esporas otorgan información relevante, sino también el tamaño de las esporas y la cantidad de esporas por esporangio, caracteres asociados a la poliploidía y al tipo de reproducción sexual o apogámica (Knobloch, 1966; Tryon \& Tryon, 1982; Tryon \& Lugardon, 1991). Estudios palinológicos realizados en $A$. nivea caracterizan a la ornamentación de las esporas por su exosporio psilado y perisporio crestado o crestado-reticulado (Tryon \& Lugardon, 1991; Morbelli et al., 2001). Las dimensiones de las esporas difieren entre las variedades y aún en la misma variedad, la var. nivea y var. tenera registran las mayores dimensiones (Hernández et al., 2015; Morbelli et al. 2001).

Algunos aspectos morfológicos de los gametofitos de Argyrochosma fueron descritos por Woronin (1907), Nayar \& Bajpai (1963) y Gabriel y Galán (2011) este último autor, aunque no indica la variedad de $A$. nivea estudiada, podría referirse a la var. nivea por la descripción morfológica que realiza sobre el esporofito; los mismos autores citan a la reproducción apogámica como frecuente en el género. La apogamia permite acortar el período de tiempo entre la espora y el esporofito (Gastony \& Windham, 1989). Este tipo de reproducción en general se presenta en plantas que habitan en ambientes con marcada estacionalidad, especialmente de ambientes xéricos, y facilita el cierre de su ciclo de vida en corto período de tiempo ante condiciones extremas por falta de agua.

Este trabajo tiene la finalidad de describir características de la fase gametofítica con el objeto de caracterizar esta fase del ciclo de vida de Argyroschoma nivea y aportar evidencias a las relaciones entre las tres variedades de la especie.

\section{Materiales y métodos}

Los ejemplares utilizados para este trabajo provienen de Argentina, según se detalla a continuación: Argrychosma nivea var. flava. Salta. Dpto. Guachipas, Cebilar, 04-02-10, Leg. Hernández et al. 1133 (LIL); 22-11-10, Leg. Hernández et al.1260 (LIL).Tucumán. Dpto. Trancas, Vipos, 05-06-12, Leg. Delgado 90 (LIL); Norte de Trancas (límite con el Tala), 06-04-2013, Leg. Delgado 404 (LIL).

Argrychosma nivea var. tenera. Salta. Dpto Cachi. Quebrada de Escoipe, 20-05-10, Leg. Hernández et al. 1300 (LIL). Tucumán. Tafi del Valle, Infiernillo, 10-04-2015, Leg. Hernández et al. 3471 (LIL). Dpto Trancas, Hualinchay, camino a Lara, 16-12-11, Leg. Hernández et al. 2084 (LIL).

Argrychosma nivea var. nivea. Salta. Dpto. Guachipas, Cebilar, 22-11-10, Leg. Hernández et al. 1261 (LIL). Tucumán. Tafi del Valle, Infiernillo, Leg. Hernández et al. 3470 (LIL). Jujuy. Dpto. Tilcara, Tilcara, 18-05-2012, Leg. Hernández et al. 2522 (LIL).

Para determinar la cantidad de esporas por esporangio, se consideraron al menos 20 cápsulas por cada especie y el valor del diámetro polar (DP) y el diámetro ecuatorial (DE) resultó de al menos 20 esporas de cada ejemplar estudiado, los resultados se expresan promedio y entre paréntesis el menor y el mayor valor.

Las esporas obtenidas para la siembra fueron previamente desinfectadas con hipoclorito de sodio al $10 \%$, durante cinco minutos en agitación continua. La siembra se llevó a cabo bajo cámara de flujo laminar, en cápsulas de petri de $5 \mathrm{~cm}$ de diám. con medio de cultivo Dyer gelificado con 9 $\mathrm{g} / \mathrm{L}$ de agar Brittania. Los cultivos se mantuvieron en cámara con ocho horas de luz.

Se realizaron observaciones periódicas cada tres o cuatro días durante tres meses después de la siembra para evaluar el desarrollo. Se fijaron ejemplares de gametofitos en hidrato de cloral acetocarminado (Edwards \& Miller, 1972) para su observación con microscopio de luz (MO), para las observaciones con microscopía electrónica de barrido (MEB) los gametofitos fueron fijados en glutaraldehído al $2 \%$ en buffer fosfato, durante 72 hs., deshidratados en una serie de alcohol etílico,

\begin{tabular}{|l|l|l|l|}
\hline $\begin{array}{l}\text { Argrychosma } \\
\text { nivea }\end{array}$ & $\mathrm{E}$ & $\mathrm{DE}(\mu \mathrm{m})$ & $\mathrm{DP}(\mu \mathrm{m})$ \\
\hline var. flava & 32 & $57,8(51-64)$ & $54,6(47-59)$ \\
\hline var. nivea & 32 & $69,77(62,9-74,2)$ & $70,5(69,3-71,7)$ \\
\hline var. tenera & 32 & $74,5(63-85)$ & $65(57-73)$ \\
\hline
\end{tabular}

Tabla 1. Cantidad de esporas por esporangio (E) y dimensiones de las esporas (DP: diámetro polar, DE: diámetro ecuatorial). 
llevados a punto crítico con dióxido de carbono y colocados sobre cinta doble faz, y finalmente cubiertos con una película de oro.

Las observaciones fueron realizadas con microscopio Olympus CX41 con cámara digital
Mshot (MC30) y con microscopio electrónico JEOL Modelo JSM 6480 LV SEM (Japan) del Laboratorio de Microscopía Electrónica de Barrido y Microanálisis (LASEM) de la Universidad Nacional de Salta.

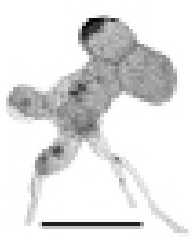

(A)
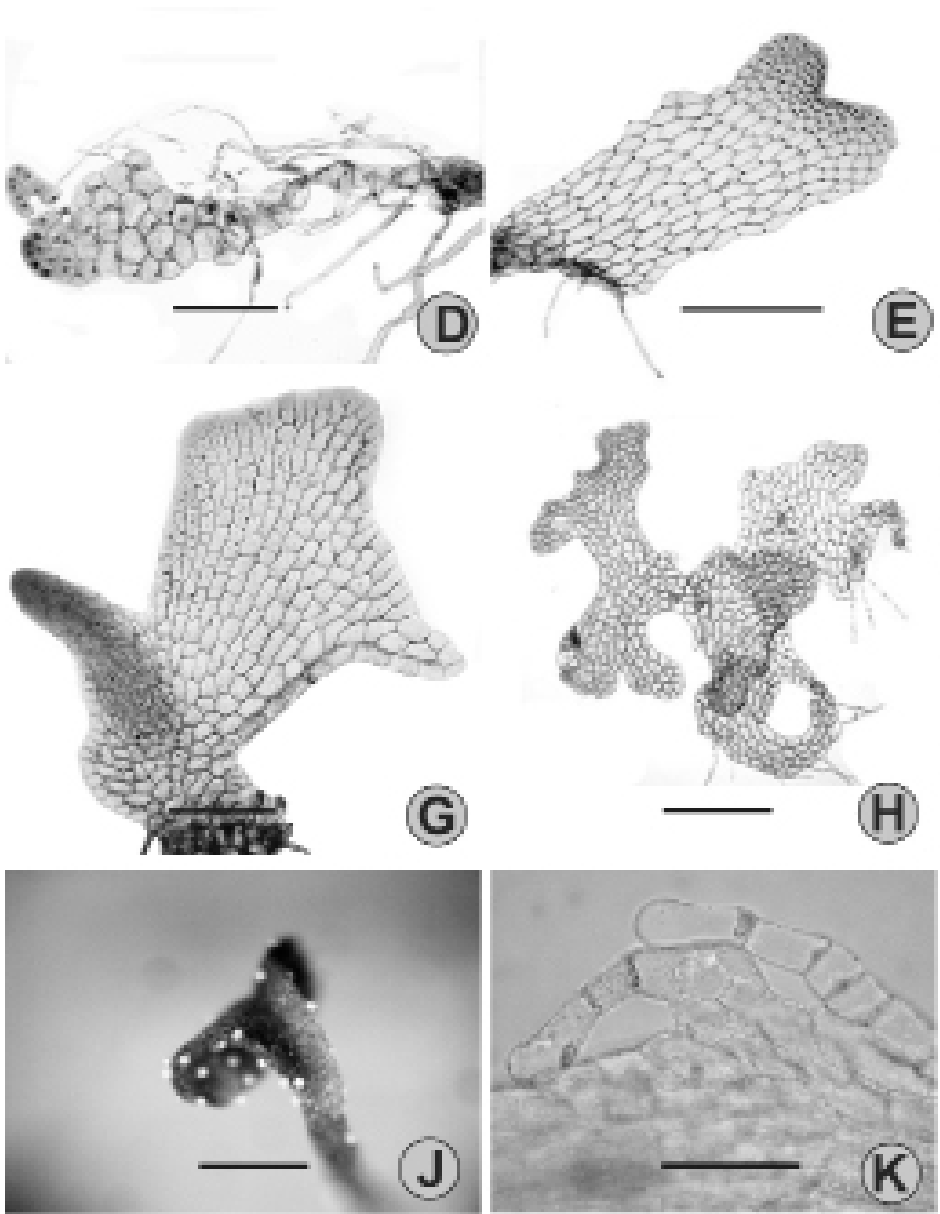

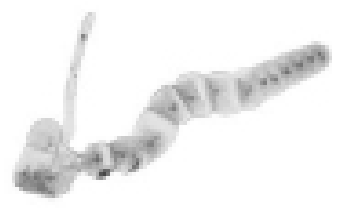

(B)
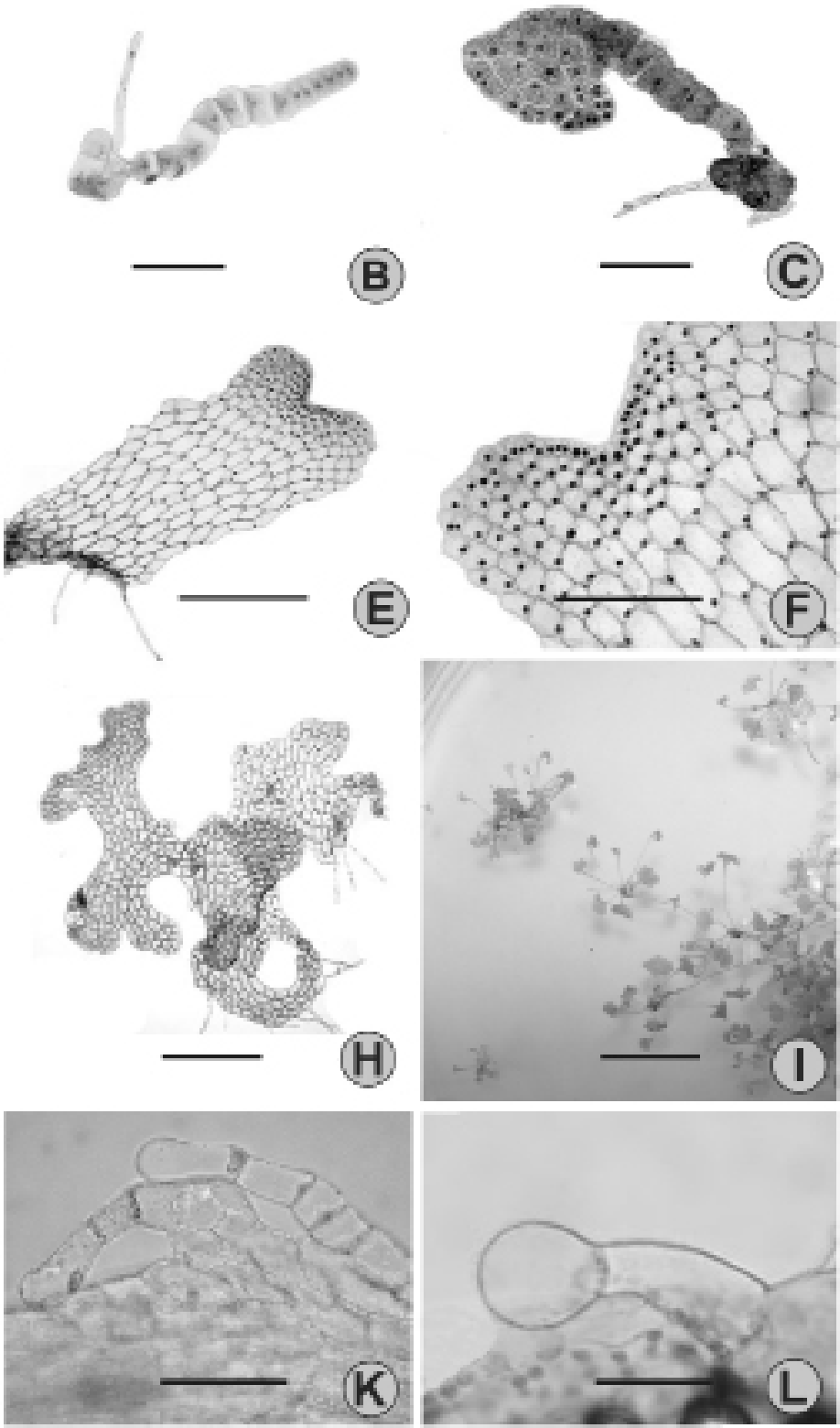

Figura 1. Desarrollo gametofitico de Argyrochosma nivea (MO). A: gametofito ramificado (var. tenera), B: gametofito filamentoso uniseriado (var. nivea), C: gametofito laminar asimétrico (var. tenera), D: gametofito laminar asimétrico (var. nivea), E: gametofito laminar cordiforme alargado (var. nivea), F: cuello de gametofito con meristemo (var. nivea), G: gametofito laminar con alas asimétricas (var. flava), H: gametofito laminar con alas irregulares (var. flava), I: esporofitos jóvenes (var. flava), J: esporofito con tricomas productores de farina (var. flava), K: tricoma pluricelular (var. flava), L: tricoma glandular (var. flava). Escalas: $\mathrm{A}, \mathrm{F}=100 \mu \mathrm{m} ; \mathrm{B}=150 \mu \mathrm{m} ; \mathrm{C}, \mathrm{D}, \mathrm{G}=200 \mu \mathrm{m} ; \mathrm{E}=300 \mu \mathrm{m} ; \mathrm{H}, \mathrm{J}=500 \mu \mathrm{m} ; \mathrm{I}=1 \mathrm{~cm}$; $\mathrm{K}, \mathrm{L}=50 \mu \mathrm{m}$. Figure 1. Gametophytic development of Argyrochosma nivea (MO). A: branched gametophyte (var. tenera), B: uniseriate filamentous gametophyte (var nivea), C: asymmetrical laminar gametophyte (var. tenera), D: asymmetrical laminar gametophyte (var. nivea), F: gametophyte neck with meristem (var nivea), G: laminar asymmetric gametophyte (var flava), H: laminar gametophyte with irregular wings (var flava), I: Young sporophytes (var. flava), J: Sporophytes with trichomes that produce farina (var. flava), K: pluricelular trichomes (var flava), L: glandular trichome (var flava). 


\section{Resultados}

\section{Esporas}

En Argyrochosma nivea el números de esporas por esporangio es de 32, y el promedio del tamaño de las esporas de es de $D E=67,51$ y $D P=61,21$ $\mu \mathrm{m}$. El número de esporas por esporangio y las dimensiones de las esporas para cada una de las variedades se presentan en la Tabla 1.

\section{Desarrollo Gametofítico}

La primer fase de desarrollo gametofítico, la germinación de esporas, comenzó en las tres variedades entre los 3 y 5 días después de realizada la siembra. Según la propuesta de Nayar \& Kaur (1971) la germinación es tipo Polar y corresponde al tipo Vittaria, donde la primer célula protálica se divide en sentido perpendicular al rizoide.

La célula protálica se divide 2 ó 3 días después de la germinación de las esporas para originar al gametofito filamentoso pluricelular. Los filamentos presentan 5-7 células en las variedades flava y tenera, y 6-12 células en la var. nivea; generalmente los gametofitos son uniseriados, excepto en la var. tenera cuyos filamentos se bifurcan en la base (figs. $1 \mathrm{~A}, \mathrm{~B})$.

Después de la fase filamentosa, entre los 7 y 10 días posteriores a la germinación, se desarrollan los gametofitos laminares jóvenes asimétricos (figs. $1 \mathrm{C}, \mathrm{D}$ ), o simétricos con un meristema apical o lateral responsable de la formación de las alas de los gametofitos maduros (figs. 1 E,F). En las variedades nivea y flava se desarrollan gametofitos con cuello y región meristemática delimitada, de aspecto cordiforme, asimétricos, con alas de diferentes proporciones (fig. 1 G) o lámina con lóbulos irregulares, sin cuello delimitado, ni región meristemática evidente (fig. $1 \mathrm{H}$ ); y en la var. tenera se origina un solo tipo de gametofitos, cordiformes asimétricos con las alas de diferentes dimensiones. Los gametofitos cordiformes a diferencia de los irregulares, presentan una costilla central pluriestratificada.

Los gametofitos son glabros durante todas las fases de su desarrollo en las tres variedades.

\section{Sexualidad de los gametofitos}

Los gametofitos maduros de las variedades nivea y tenera son neutros o vegetativos, mientras que los gametofitos de la var. flava son diocos o unisexuales. Los gametangios masculinos se forman en la etapa laminar madura, su cantidad es muy baja, entre 3 y 8 anteridios distribuidos al azar. Los gametangios femeninos se originan sobre la costilla central del gametofito y la cantidad de arquegonios es de 7 y 15 unidades por gametofito maduro.
La estructura de los gametangios corresponde a la clásica de los helechos leptosporangiados, los anteridios con tres células (basal, anular y opercular) y los arquegonios con cuatro columnas de cuatro células cada uno.

\section{Esporofitos}

La formación de los esporofitos comienza a evidenciarse a partir de los 40-50 días desde la germinación de las esporas. En las tres variedades los esporofitos son de origen apogámico, en las variedades flava y tenera se desarrollan a partir de mamelones meristemáticos cubiertos de escamas (figs. 2 A-C) que se originan sobre la costilla del gametofito, y en la var. nivea la costilla central se extiende por encima de la superficie para originar el esporofito con tricomas en la base (figs. 2 D,E). En las variedades nivea y tenera los esporofitos son glabros, y en la var. flava se originan entre 5 y 10 esporofitos por gametofito, las plántulas presentan tricomas 3-4-celulares y bicelulares glandulares productores de farina de color blanco (figs. 1 I-L, 2 F-I), distribuidos sobre el peciolo y en ambos lados de la lámina: los tricomas glandulares se evidencian cuando las plántulas toman contacto con el aire, mientras los esporofitos permanecen sumergidos en el medio de cultivo gelificado, no producen farinas.

\section{Discusión y conclusión}

Las esporas de Argyrochosma nivea, en sus tres variedades, tienen dimensiones semejantes a las descritas por Morbelli et al. (2001), y la cantidad de esporas por esporangio es uniforme en la especie, dato correspondiente con el registro de Gabriel y Galán (2011) y Hernández et al. (2015). Las dimensiones de las esporas, en las tres variedades, encontradas en este trabajo son menores que las citadas por Morbelli et al (2001) y Hernández et al. (2015). La variedad nivea se destaca por la amplia variación en el tamaño de las esporas, con DE= 38,3-106 (Morbelli et al., 2001), semejante observación se encuentra en la var. tenera, con DE= 37-106 (Hernández et al., 2015).

El modelo de germinación de las esporas, tipo Vittaria, observado en el material estudiado es frecuente en los helechos cheilantoideos (Nayar \& Kaur, 1971) y el tiempo de germinación es breve, posiblemente porque las esporas sembradas no tuvieron previo almacenamiento.

En los gametofitos filamentosos, la longitud no es un carácter constante, sin embargo la cantidad de células encontradas en la var. nivea es similar a la observada en los gametofitos provenientes de plantas colectadas en Perú citadas por 

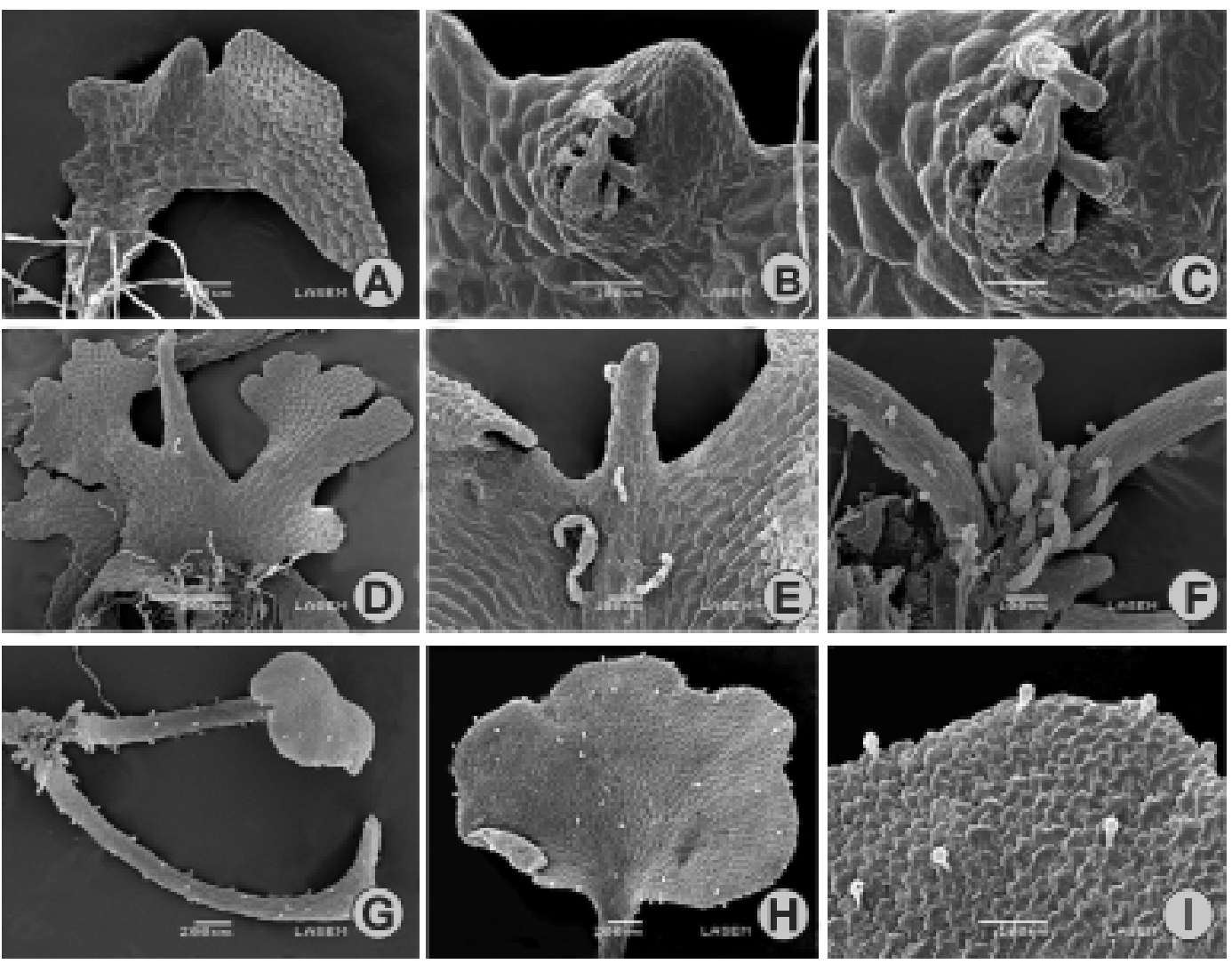

Figura 2. Gametofitos y Esporofitos de Argyrochosma nivea (MEB). var. tenera. A: gametofito con alas asimétricas, B: gametofito con mamelón, $\mathbf{C}$ : detalle de mamelón con escamas. var. nivea. D: gametofito laminar con alas asimétricas, E: gametofito con tricomas pluricelulares. var. flava. F: esporofitos jóvenes, G: esporofitos con tricomas glandulares, $\mathbf{H}$ : lámina de esporofito con tricomas glandulares, I: detalle de tricomas glandulares. Figure 2. Gametophytes and Sporophytes of Argyrochosma nivea. (MEB). var. tenera. A: gametophyte with asymmetrical wings, B: cushion on gametophyte, $\boldsymbol{C}$ : cushion with scales. var. nivea. D: laminar gametophyte with asymmetric wings, $\boldsymbol{E}$, gametophyte with pluiricellular trichomes. var. flava. F: young sporophytes, $\mathbf{G}$ : sporophytes with glandular trichomes, $\boldsymbol{H}$ : sporophyte leaf with glandular trichomes, I: detail of glandular trichomes.

Gabriel y Galán (2011); la ramificación de los gametofitos filamentosos influye en la morfología de los gametofitos maduros, así por ejemplo especies del género Pteris desarrollan gametofitos maduros individuales o agrupados en rosetas, correspondientes a gametofitos uniseriados o ramificados respectivamente (Martínez et al. 2013), en este caso se observa que los gametofitos filamentosos de A. nivea var. tenera se diferencian de las otras dos variedades porque las ramificaciones de los gametofitos filamentosos desarrollan gametofitos laminares independientes que se mantienen unidos en la base en etapas posteriores.

Durante el desarrollo de la lámina del gametofito en Argyrochosma nivea predomina el tipo Ceratopteris, excepto la var. nivea que presenta dos modelos, tipo Adiantum y Ceratopteris, ambos frecuentes en otros helechos cheilantoideos (Nayar \& Kaur, 1971). La forma dominante de los gametofitos maduros es cordada asimétrica, los gametofitos irregulares, con láminas profundamente escotadas de la var. nivea coinciden con los registros de Gabriel y Galán (2011) y también fueron observados en otras Pteridaceae, como Pteris ensiformis Burm. f., una especie poliploide (Martínez 2010, Walker 1962).

Los gametofitos son glabros durante todo su desarrollo a pesar que los esporofitos de las variedades nivea y flava presentan farinas en el envés de sus láminas, a diferencia de otros helechos con farinas como las especies de Notholaena donde los tricomas glandulares productores de farinas están presentes en ambas fases del ciclo de vida (Johnson et al. 2012). Sigel et al. (2011) al estudiar las relaciones filogenéticas de las especies con farinas, encuentran que la farina del género Argyrochosma surgió de manera independiente de la farina de las especies del género Notholaena.

La expresión sexual de los gametofitos adultos se manifiesta en la var. flava, donde a pesar de 
generar gametofitos dioicos, los esporofitos son apogámicos al igual que en las otras variedades, la apogamia es frecuente en el género Argyrochosma y otros helechos cheilantoideos (Hayes, 1924; Knobloch, 1966; Steil, 1933; Whittier, 1965, 1970). Este tipo de reproducción se encuentra relacionado con los niveles de ploidía y el tamaño de las esporas (Knobloch, 1966, 1969). En las plantas estudiadas, la cantidad de esporas es de 32, y el número básico de cromosomas es $x=27$ (Windham \& Yatskievych, 2003); según Hernández et al. (2015) y Sigel et al. (2011) las variedades nivea y tenera son triploides con $2 \mathrm{n}=81$, y aunque se desconoce el número cromosómico de la var. flava, según el tamaño de sus esporas, es probable su ploidía, todo esto explica el origen apogámico de los esporofitos.

Los esporofitos de la var. flava, se caracterizan por la abundancia de tricomas productores de farina blanca, diferente a las plantas maduras que se distinguen por su farina amarilla. Sigel et al. (2011) sugieren que podría haber disparidades químicas relacionadas con la funcionalidad en las diferentes fases del ciclo de vida de las plantas. La composición química de la farina ha demostrado ser un importante carácter filogenético entre los diferentes grupos taxonómicos de los helechos cheilantoideos (Wollenweber, 1984; Wollenweber \& Schneider, 2000; Sigel et al., 2011).

Se concluye que las tres variedades de Argyrochosma nivea (var. flava, var. nivea y var. tenera) comparten los siguientes caracteres relacionados con la fase gametofítica: número de esporas $=32$, tipo de germinación tipo Vittaria, formación de gametofitos cordiformes asimétricos, con alas de diferentes proporciones, y reproducción apogámica. Si bien las variedades nivea y flava presentan farinas, únicamente la variedad flava desarrolla esporofitos jóvenes con tricomas productores de farinas.

\section{Agradecimientos}

A la Fundación Miguel Lillo y Consejo de Investigación de la Universidad Nacional de Salta (U.N.Sa.) por el financiamiento para realizar este trabajo, y al servicio técnico del Laboratorio de microscopía electrónica de barrido y microanálisis de la U.N.Sa. por la asistencia personalizada.

\section{Referencias}

Edwards, M.E. \& Miller, J.H. (1972). Growth regulation by ethylene in fern gametophytes. III. Inhibition of spore germination. Amer. J. Bot. 59: 458-465.

Gabriel y Galán, J. M. (2011). Gametophyte development and reproduction of Argyrochosma nivea
(Pteridaceae). Biologia 66(1): 50-54

Gastony, G.J. \& Windham, M.D. (1989). Species concepts in Pteridophytes: the treatment and definition of agamosporous species. Amer. Fern J. 79: 65-77.

Hernández, M.A., Andrada, R.A., Páez, V. A. \& Martínez, O.G. (2015). Ploidy level and obligate apogamy in Argyrochosma nivea (Poir.) Windham var. tenera (Pteridaceae). Hoehnea 42(2): 233-237.

Hayes, D.W. (1924). Some Studies of Apogamy in Pellaea atropurpurea. (L.) Link. Transact. Microsc. Soc. 43(3): $119-135$.

Hevly, R.H. (1963). Adaptations of cheilanthoid ferns to desert environments. J. Arizona Acad. Sci. 2: 164-175.

Johnson, A.K., Rothfels, C.J., Windham, M.D. \& Pryer, K.M. (2012). Unique expression of a sporophytic character on the Gametophytes of notholaenid ferns (Pteridaceae). Amer. J. Bot. 99(6): 1118-1124.

Knobloch, I.W. (1966). A preliminary review of spore number and apogamy within the genus Cheilanthes. Amer. Fern J. 56(6):163-167.

Knobloch, I.W. (1969). The spore pattern in some species of Cheilanthes. Amer. J. Bot. 56(6): 646-653.

Martínez, O.G. (2010). Gametófitos y esporófitos jóvenes de cuatro especies naturalizadas de Pteris (Pteridaceae) en América. Rev. Biol. Trop. 59(1): 8192.

Martínez, O.G., C. Prada, Tanco, M.E. \& Bonomo, M.C. (2013). Sexual phase of three species of Pteris (Pteridaceae). Trop. Plant Biol. 6(1):46-52.

Morbelli, M.A., Ponce, M.M., Macluf, C. \& Piñeiro, M.R. (2001). Palynology of South American Argyrochosma and Notholaena (Pteridaceae) species. Grana, 40: 6, 280-291.

Nayar, B.K. \& Bajpai, N. (1963). Morphology of the gametophytes of some species of Pellaea and Notholaena. J. Linn. Soc. Bot. 59: 63-76.

Nayar, B.K. \& Kaur, S. (1971). Gametophytes of homosporous ferns. Bot. Rev. 37: 295-396.

Ponce, M.M. (2016). Argyrochosma (J. Sm.) Windham, in: Zuloaga F.O. \& M.J. Belgrano \& A.M. Anton (eds.). Flora vascular de la República Argentina II. pp. 285287.

Sigel, E.M., Windham, M.D. Huiet, L. Yatskievych, G. \& Pryer, K.M. (2011). Species relationships and farina evolution in the cheilanthoid fern genus Argyrochosma (Pteridaceae). Syst. Bot. 36: 554-564.

Steil, W. N. (1933). New Cases of Apogamy in Certain Homosporous Leptosporangiate Ferns. Bot. Gaz. 95 (1): 164-167.

Tryon, R.M., Tryon, A.F. Kramer, K.U. (1990). Pteridaceae, in: Kubitzki K. (ed.). The families and genera of vascular plants. Berlin: Springer-Verlag. I: 230-256.

Tryon, A. F. \& Lugardon, B. (1991). Spores of the Pteridophyta. Springer, New York.

Tryon, R.M. \& Tryon, A.F. (1982). Ferns and allied plants, with special reference to Tropical America. SpringerVerlag. New York.

Walker, T.G. (1962). Cytology and evolution in the fern genus Pteris L. Evolution 16: 27-43.

Windham, M.D. \& Yatskievy, G. (2003). Chromosome studies of cheilanthoid ferns (Pteridaceae: 
Cheilanthoideae) from the western United States and Mexico. Amer. J. Bot. 90: 1788-1800.

Windham, M.D. (1987). Argyrochosma, a new genus of cheilanthoid ferns. Amer. Fern J. 77: 37-41.

Whittier, D.P. (1965). Obligate apogamy in Cheilanthes tomentosa and C. alabamensis. Bot. Gaz. 126 (4): 275-281.

Whittier, D.P. (1970). The initiation of sporophytes by obligate apogamy in Cheilanthes castanea. Am. J.
Bot. 57(10): 1249-1254.

Wollenweber, E. \& Schneider, D. H. (2000. Lipophilic exudates of Pteridaceae-Chemistry and chemotaxonomy. Biochem. Syst. Ecol. 28: 751-777.

Wollenweber, E. (1984). Exudate flavonoids of Mexican ferns as chemotaxonomic markers. Revista Latinoamer. Quím. 15: 3-11.

Woronin, H. (1907). Apogamie und aposporie bei einigen farnen. Ber. Deutsch. Bot. Ges. 25: 115-135. 
